\title{
Response to the letter of Hoermann and colleagues
}

\section{Robin P Peeters $\mathbb{1}$ and Juan P Brito ${ }^{2}$}

${ }^{1}$ Department of Internal Medicine, Erasmus MC Academic Center for Thyroid Disease, Erasmus MC, Rotterdam, The Netherlands and ${ }^{2}$ Mayo Clinic, Knowledge and Evaluation Research Unit in Endocrinology (KER_Endo), Division of Endocrinology, Diabetes, Metabolism and Nutrition, Department of Medicine, Rochester, Minnesota, USA

Correspondence should be addressed to R P Peeters

Email

r.peeters@erasmusmc.nl
We read the letter by Hoermann and colleagues with great interest and are thankful for the opportunity to discuss some of their remarks (1).

The authors raise the issue that part of the controversy regarding the treatment of subclinical hypothyroidism $(\mathrm{SCH})$ is related to how we define $\mathrm{SCH}$ and whether it is a true disease or merely a laboratory constellation.

Although we wrote a debate (2) on whether SCH should be treated or not, we generally agree that the biochemical diagnosis of SCH covers a wide spectrum of patients. We, therefore, agree that $\mathrm{SCH}$ can be a true disease in one patient but a laboratory constellation in another. Based on the progression rate to overt hypothyroidism, the extent of TSH elevation is a good discriminator between the two (3, 4). Consequently, we have a consensus on the treatment of $\mathrm{SCH}$ in the vast majority of cases. For example, in the case of a very mildly elevated TSH, and FT4 above the median and negative TPO-antibodies (case A), we both agreed not to start with levothyroxine (LT4) therapy, whereas in the case of a clearly elevated TSH, positive TPO-antibodies and a low-normal FT4 (case B) we both agreed to start with LT4 therapy (2). However, as always in medicine, there is a grey zone, such as when $\mathrm{SCH}$ is diagnosed based on a TSH between 7 and $10 \mathrm{mIU} / \mathrm{L}$ and nonspecific symptoms. Unfortunately, adequately powered clinical trials with patients in this grey zone are lacking. For these patients, we fully agree with Hoermann and colleagues that the measurement of only a TSH cannot reliably discriminate between true disease or laboratory variation. This uncertainty about SCH diagnosis and its association with patient symptomatology has confused patients and clinicians in how to best address it. Hoermann and colleagues proposed a strategy that relies more on clinical manifestations (e.g. symptoms of hypothyroidism) and less on TSH values. The fact the hypothyroidism symptoms are nonspecific (5) suggests that this approach will not be able to provide a clear-cut answer either. Alternatively, we see this grey zone as an opportunity to bring the uncertainty about SCH diagnosis and treatment in the conversation with the patient to find the best strategy that responds to the situation. Sometimes the clinician's response demands focusing on addressing symptoms (e.g. investigate other etiologies of fatigue) as opposed to lowering TSH values. At other times, the clinician's response could be a therapeutic trial with the LT4 to normalize TSH and patient re-assessment for symptom change or resolution. Future research will provide more clarity about the diagnosis of $\mathrm{SCH}$ and the effectiveness of treatment. In the meantime, we should welcome the uncertainty of SCH diagnosis and treatment as an opportunity to work together with our patients and uncover what is best for them.

Declaration of interest

Dr Peeters has received lecture fees from IBSA and Merck. Dr Brito reports no conflicts of interest.

\section{Funding}

This research did not receive any specific grant from any funding agency in the public, commercial or not-for-profit sector.

\section{References}

1 Hoerman R, Midgley J, Larisch R \& Dietrich J. Treatment options for subclinical hypothyroidism. European Journal of Endocrinology 2021 185 L5-L6. (https://doi.org/10.1530/EJE-20-1405).

2 Peeters RP \& Brito JP. Subclinical hypothyroidism: to treat or not to treat? European Journal of Endocrinology 2020183 D15-D24. (https:// doi.org/10.1530/EJE-20-0621)
Published by Bioscientifica Ltd. 
3 Diez JJ, Iglesias P \& Burman KD. Spontaneous normalization of thyrotropin concentrations in patients with subclinical hypothyroidism. Journal of Clinical Endocrinology and Metabolism 2005 90 4124-4127. (https://doi.org/10.1210/jc.2005-0375)

4 Huber G, Staub JJ, Meier C, Mitrache C, Guglielmetti M, Huber P $\&$ Braverman LE. Prospective study of the spontaneous course of subclinical hypothyroidism: prognostic value of thyrotropin, thyroid reserve, and thyroid antibodies. Journal of Clinical Endocrinology and Metabolism 200287 3221-3226. (https://doi.org/10.1210/jcem.87.7.8678)

5 Canaris GJ, Manowitz NR, Mayor G \& Ridgway EC. The Colorado thyroid disease prevalence study. Archives of Internal Medicine 2000160 526-534. (https://doi.org/10.1001/archinte.160.4.526)

Received 29 June 2021

Accepted 6 July 2021 